\title{
UN PROGRAMA PARA EL FORTALECIMIENTO FAMILIAR: ALTERNATIVA AL MALTRATO DE MENORES
}

Dra. María M. Lizardi, Catedrática

\section{Descripción del Proyecto}

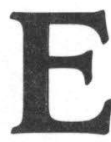

1 Proyecto de Apoyo Familiar a Niños y Adultos, mejor conocido como AFANA-HOMEBUILDERS, se estableció en la ciudad de Mayagüez el $1^{\text {ro. }}$ de octubre de 1996, como una respuesta creativa e innovadora al problema de maltrato de menores en dicha ciudad. Dicho proyecto representa el resultado del esfuerzo conjunto del Departamento de la Familia, Administración de Familias y Niños del Instituto Individual Group and Organizational Development Inc., una organización sin fines de lucro. El Proyecto incorpora en su base programática tres componentes básicos: servicio directo, adiestramiento e investigación.

El proyecto reconoce los efectos devastadores del maltrato de menores en el desarrollo físico, social y emocional de los niños. El niño, en su desarrollo socio-emocional, requiere de una relación significativa con sus padres o encargados. Muchas veces ese desarrollo se ve afectado, ya que la relación está basada en el maltrato del cual el niño es objeto. El escenario en el cual generalmente se comete abuso contra un menor es el hogar y son los familiares, particularmente los padres, los ejecutadores del mismo.

El maltrato a menores en Puerto Rico tiene varias manifestaciones que están definidas en la Ley 75 del 20 de mayo de 1980. Éstas son: abuso físico, emocional, sexual, negligencia, abandono y explotación. Lamentablemente, en Puerto Rico va en aumento el número de niños maltratados física, emocional y sexualmente por sus 
progenitores o encargados. Estadísticas de los años 1992 al 1995 presentadas por el Departamento de la Familia, reflejan dicho aumento:

\section{TABLA I}

Situaciones de maltrato de menores referidos al Departamento de la Familia y número de familias activas de 1992 al 1995

\begin{tabular}{ccc}
\hline Años & $\begin{array}{c}\text { Referidos } \\
\text { (Querellas al DF) }\end{array}$ & Activos \\
\hline $1992-93$ & 65,690 & 37,905 \\
$1993-94$ & 76,923 & 43,381 \\
$1994-95$ & 92,934 & 48,705 \\
\hline
\end{tabular}

La región de Mayagüez es una de las más afectadas por el maltrato de menores y menos provista de servicios de urgencia que ameritan estas familias. Las estadísticas reflejan los siguientes datos:

\section{TABLA II}

Situaciones de Maltrato de menores referidos al Departamento de la Familia y número de familias activas de la región de Mayagüez de 1992 al 1995

\begin{tabular}{ccc}
\hline Años & $\begin{array}{c}\text { Referidos } \\
\text { (Querellas } \\
\text { al DF) }\end{array}$ & $\begin{array}{c}\text { Activos } \\
\text { (Niños } \\
\text { Afectados) }\end{array}$ \\
\hline $1992-93$ & 6,060 & 5,373 \\
$1993-94$ & 4,886 & 5,189 \\
$1994-95$ & 6,871 & 5,766 \\
\hline
\end{tabular}

La situación planteada presentaba la necesidad de trabajar con las familias utilizando otros acercamientos de intervención de manera que se redujera el impacto negativo de la violencia en el seno familiar. Para responder a dicha necesidad se implantó el Proyecto Demostrativo conocido como AFANA-HOMEBUILDERS en la ciudad de Mayagüez. 
Las metas principales del Proyecto son:

- proteger a los niños

- mantener y fortalecer los lazos familiares

- estabilizar la situación de crisis en familias que incurren en maltrato de menores

- aumentar las destrezas y competencias de la familia

- facilitar el acceso de la familia hacia una variedad de sistemas de apoyo formales e informales

- prevenir la remoción del menor de su hogar en la medida que sea posible. (E. Tracy, D. Haapala y P. Pecora, 1991)

Aunque los servicios de fortalecimiento familiar están relacionados a otros servicios centrados en la familia y servicios con base en el hogar en filosofía, justificación y origen, éstos son diferentes en sus estrategias y componentes de servicio.

\section{Prestación de servicios}

El Proyecto AFANA-HOMEBUILDERS de la ciudad de Mayagüez está basado teóricamente en el modelo de intervención con familias conocido como "Constructores de hogares" o "Homebuilders" por su nombre en inglés. Éste representa una respuesta estratégica a las familias en crisis que incurren en maltrato de menores. Este modelo fue desarrollado en el 1974 en Tacoma, Washington bajo los auspicios de la organización Servicios Comunitarios Católicos (Kinney, Haapala, Booth y Leavitt, 1990).

El propósito del modelo es evitar tener que remover a un menor de su hogar cuando existen problemas de abuso y maltrato de menores.

El modelo presenta las siguientes características:

- Se aceptan sólo familias en las cuales existe un riesgo inminente de remoción de un menor del hogar.

- Los servicios están orientados hacia la intervención en crisis. Las familias son atendidas por los/as trabajadores/as sociales del Proyecto dentro de las 24 horas de ser referidas. 
- El personal de servicio está accesible todo el tiempo por lo cual se mantiene un programa flexible los siete días de la semana.

- Los procesos de intervención y ponderación de la situación aseguran que los niños no se dejarán en situaciones de peligro.

- Aunque se atienden problemas de los componentes del sistema familiar, el enfoque del modelo está dirigido a la familia y su contexto. No se concibe a padres/madres e hijos/as o encargados/as desde una perspectiva de patología familiar, sino desde una perspectiva de salud, fortaleza y potencial hacia el cambio.

Los/as trabajadores/as sociales visitan a las familias en su hogar, vecindario y comunidad. La frecuencia de las visitas dependerá de las necesidades de la familia. Se entiende que la intervención debe ocurrir en el hábitat natural de la familia, ya que es allí donde ocurren las transacciones familiares. De esta manera se rompe con el esquema tradicional del trabajo social en el cual los servicios se ofrecen en una oficina o contexto organizacional.

El modelo combina la enseñanza de destrezas para cambiar conductas, el enseñar a la familia cómo obtener los recursos y servicios necesarios y la consejería basada en un entendimiento de cómo establecer una relación de ayuda empática que motive al/la cliente hacia el cambio esperado.

Los servicios están basados en las necesidades identificadas por el/la trabajador/a social y la familia

La participación de las familias es voluntaria y los acuerdos de trabajo se formalizan en un plan de acción o contrato elaborado con la participación activa de la familia. De esta manera se obtiene un compromiso hacia el cambio y se reconoce la capacidad de autodeterminación de los/las participantes.

La carga de situaciones del/la trabajador/a es limitada; por lo general se trabaja con dos familias al mismo tiempo

Ésta es una de las diferencias mayores que propone el modelo y 
la que despierta mayor controversia. Sin embargo, el trabajar con un número reducido de situaciones es lo que permite que el profesional de ayuda pueda tener intervenciones más frecuentes e intensas logrando cambios en un período más corto de tiempo.

El periodo en el cual se trabaja con la familia es breve con un promedio de cuatro a seis semanas de duración

Esta característica es complementaria a la carga reducida de situaciones familiares con las cuales se interviene. También es cónsona al acercamiento de intervención en crisis que propone el modelo.

Se da más énfasis a las fortalezas y recursos de la familia que a las limitaciones

El modelo se aparta de la dicotomía de salud/enfermedad y percibe al ser humano en un continuo de bienestar social que, en diferentes circunstancias o transiciones de vida, puede fluctuar. Se entiende que en la medida que trabajamos movilizando las fortalezas y recursos internos y externos de la familia, se fomentan las capacidades adaptativas de la familia frente a las demandas que presenta su entorno social.

El/la trabajador/a social lleva a cabo una serie de roles profesionales tales como: terapeuta, consejero/a, educador/a intercesor/a, consultor/a y proveedor/a de servicios.

\section{Bases filosóficas del proyecto}

El modelo está basado en una serie de postulados filosóficos, los cuales se presentarán a continuación:

En la mayoria de las situaciones es mejor para los niños crecer en sus familias naturales

Se entiende que aun cuando haya emociones confusas e interacciones dolorosas, también existe en las familias amor, preocupación y sentido de pertenencia. Estos sentimientos pueden aflorar y crecer si se ayuda a la familia a canalizar sus problemas de una forma socialmente aceptable y no a través de la violencia física o verbal. Se parte de la premisa de que es mejor enseñar a la familia sobre el manejo de sus problemas de vida en vez de depender del 
Estado para su solución. En la mayoría de los casos, los padres pueden aprender a establecer límites, controlar emociones y proveer para las necesidades básicas de sus hijos.

Los/as niños/as que se separan de sus familias pierden parte de la historia familiar lo cual afecta su sentido de pertenencia y continuidad. Los/as niños/as en hogares de crianza u otras instituciones pueden ser etiquetados como desviados por sus pares. También éstos/as pueden adoptar como modelos de roles a niños/as con conductas problemáticas en los hogares de grupo. Además, pierden la oportunidad de pasar por eventos significativos de la familia como celebraciones y festividades.

Los/as niños/as en albergues muchas veces son movidos de un lugar a otro, lo cual limita el establecer relaciones consistentes con figuras significativas, aun con los profesionales de ayuda. Esta falta de continuidad puede hacer difícil el establecer una identidad, sentirse importantes o planear para el futuro.

No es fácil determinar qué familia se beneficiaría más de la intervención profesional y cuál se considera que no tiene esperanza

Los autores del modelo constructores de hogares ("homebuilders") establecen que es difícil establecer de antemano el tipo de familia que se beneficiaría de la intervención profesional (Kinney, Haapala, y Booth, 1991). Por lo tanto, no descarta ningún tipo de problema en particular. Sin embargo, señalan que, en su experiencia, aquellas familias que están tan involucradas en el uso de drogas que su vida entera está enfocada en obtener la misma y en sobrevivir en culturas de drogas, son las que representan mayor peligro para sus hijos menores.

\section{El propósito del modelo es proveer esperanza a la familia}

El modelo postula que podemos proveer esperanza a la familia al minimizar barreras al cambio y hacer accesibles los servicios para que la familia acepte la intervención del/la trabajador/a social y entienda su rol. Muchas de las familias que incurren en maltrato de menores han tenido experiencias previas con profesionales de ayuda. Por eso se desarrolla un sentimiento de frustración y desesperanza, 
de que la consejería no dará resultado. Es entonces que el/la trabajador/a social debe instilar una esperanza en la familia, ampliando la perspectiva o visión del proceso de ayuda como una estrategia más amplia que la consejería y que conlleva otras alternativas de acción. Es importante que los participantes entiendan que el proceso conlleva una mutualidad de intereses y no sólo los del profesional de ayuda.

\section{Los clientes pueden aportar al proceso de ayuda}

El modelo descarta la dicotomía en la visión de las personas como saludables o enfermas. Se visualiza el funcionamiento social como un continuo en el cual cualquier persona podría necesitar ayuda alguna vez en su vida. El poder hacia el cambio nace dentro de la persona y el/la trabajador/a social interviene hacia la movilización de dichas fortalezas. Se reconoce que el cliente tiene mayor información sobre su vida que nosotros, con toda nuestra preparación profesional. Cuando el/la trabajador/a social trata a la familia con dignidad y respeto ésta responde de una forma más positiva. El compromiso del/ la profesional de ayuda se refleja en la confianza que deposita la familia en éste/a.

\section{La gente hace lo mejor que puede}

El modelo plantea que las personas hacemos lo mejor que podemos de acuerdo a la información, energía y recursos que tenemos en un momento dado. A menudo el abuso es un efecto secundario de las luchas personales con las cuales nos enfrentamos día a día. Se plantea que la gente se hiere debido a falta de información y destrezas para manejar el coraje o la creencia de que el castigo físico es el mejor método para disciplinar a un niño.

Podemos hacer daño mediante nuestra intervención, por lo tanto, debemos ser sumamente cuidadosos en este proceso

El/la trabajador/a social debe estar consciente de que no todas las técnicas de intervención son apropiadas para todas las familias. Por lo tanto, no debe ser rígido en su intervención ya que esto podría provocar mayor confusión y sentimientos de impotencia en las familias servidas. 


\section{Componentes programáticos}

Como se indicó anteriormente el Proyecto consta de tres componentes programáticos a saber: el servicio directo a la familia, adiestramiento a profesionales de ayuda e investigación.

\section{Servicio directo}

La prestación de servicios directos está a cargo de trabajadoras sociales con maestría. Éstas fueron adiestradas en el modelo HOMEBUILDERS por las personas que desarrollaron el mismo, entre ellas la señora Charlotte Booth quien es trabajadora social y Directora del Behavioral Science Institute ubicado en la ciudad de Seattle, Washington.

Los participantes son referidos por la oficina local del Departamento de la Familia. Para fines del referido de clientes se elaboró una guía la cual contiene los criterios para seleccionar a los familiares. Dichos criterios están basados en los postulados filosóficos que presenta el modelo HOMEBUILDERS.

La metodología utilizada al intervenir con las familias incluye el trabajo con individuos, la intervención con la familia como grupo y talleres de educación a padres. El servicio se presta en el hogar y la comunidad aunque las actividades educativas dirigidas a los padres $\mathrm{y}$ las actividades recreativas pueden llevarse a cabo en la oficina $u$ otros ambientes.

\section{Adiestramiento}

El Proyecto AFANA-HOMEBUILDERS tiene en su base programática un componente de adiestramiento, ya que éste se desarrolló como proyecto demostrativo con el propósito de ponderar su aplicabilidad en el contexto de la realidad puertorriqueña. Al momento se proveyó adiestramiento no sólo al personal de servicio del proyecto sino a otro personal del Departamento de la Familia.

En estos momentos se ha desarrollado un convenio colaborativo con la Universidad Pontificia Católica de Ponce para adiestrar a trabajadores sociales en el modelo. Se espera poder establecer convenios con otras universidades tanto a nivel de bachillerato como a nivel de maestría en un futuro no muy lejano. 


\section{Investigación}

El proyecto incorpora el componente de investigación en su base programática con el propósito de proveer la oportunidad de evaluar sistemáticamente la efectividad del modelo de intervención. Para esto se han diseñado formularios y procedimientos sistemáticos para la evaluación formativa del proceso y la evaluación sumativa del producto.

La evaluación contó con el insumo de las familias participantes. La misma cubrió los aspectos: programáticos, administrativos y el servicio directo. Se utilizan técnicas de carácter cuantitativo así como cualitativo. Algunas de éstas fueron: testimonios grabados de los participantes, diarios de los trabajadores sociales, lista de cotejo incluyendo metas y su logro, testimonios de los trabajadores sociales, entrevistas a otros profesionales, discusión de situaciones con el equipo de trabajo, observación directa de la familia en diversos contextos sociales y observación de los/las padres/madres mediante su participación en diferentes talleres.

Parte importante del proceso de evaluación recogía el grado de satisfacción de los/las clientes/as con el servicio recibido. Esto facilitó que el personal de servicio directo y el personal administrativo pudiera tomar las medidas necesarias para fortalecer el servicio.

\section{Fases de la intervención con las familias del modelo AFANA-HOMEBUILDERS}

El modelo HOMEBUILDERS original no presentó un detalle de las fases de intervención. La descripción de las fases a continuación representa una de las adaptaciones hechas al modelo a base de los postulados filosóficos que éste propone. El proceso de intervención consta de tres fases: la fase inicial o de contacto, la fase de trabajo intensivo y la fase de terminación.

\section{Fase inicial}

Durante la fase inicial el/la trabajador/a social explica a la familia referida los propósitos de su intervención. Es importante que la familia entienda que su participación en el programa es voluntaria. En esta fase se establece en forma conjunta con los clientes el contrato y el plan de servicios. La mutualidad de intereses del/la cliente/a 
es de singular importancia para lograr los objetivos de la intervención. Se le explica a la familia que el/la trabajador/a social estará disponible las 24 horas y se le indica la forma de comunicarse con éste/a. Se hace una exposición a la familia sobre los fundamentos filosóficos del modelo como parte del contrato inicial.

Es sumamente importante la forma en que el/la profesional de ayuda se proyecte en su intervención profesional. Debe proveer esperanza y confianza a la familia mediante un acercamiento amigable y cordial. En este sentido es más importante el acercamiento terapéutico entre él/la trabajador/a social y la familia que el tener un repertorio ilimitado de técnicas sin haber logrado una relación empática con la familia servida.

En esta primera fase él/la trabajador/a social explora y determina las necesidades del grupo familiar. Hay que tener en cuenta que al intervenir en situaciones de crisis estos procesos surgen de forma diferente.

En situaciones de crisis se procederá a poner en función los cinco componentes de la primera ayuda psicológica (Slaikeu, 1996), a saber:

- realizar el contacto psicológico

- examinar las dimensiones del problema

- analizar posibles soluciones

- apoyar la ejecución de acción concreta

- seguimiento

\section{Fase intermedia}

La fase intermedia representa el trabajo intensivo con la familia para lograr que ésta recupere el balance. Las visitas al hogar son frecuentes y pueden durar varias horas de acuerdo a las necesidades presentadas. El/la trabajador/a social puede reunirse con la familia en escenarios no tradicionales tales como un negocio de comida rápida, un parque o un centro comercial.

El/la trabajador/a social es terapeuta, educador, intercesor, facilitador de cambios, mediador y proveedor de servicios tangibles. Debido al enfoque ecológico del modelo se entiende que en la mayoría de las situaciones es necesario modificar el ambiente para lograr estimular las capacidades adaptativas de la familia en sus transacciones 
con éste. El proyecto cuenta con recursos para proveer ayuda tangible a las familias cuando ésta fuera requisito para lograr otros cambios. Estos recursos se logran a través de la coordinación entre organizaciones públicas y privadas de la comunidad, las cuales representan a los sistemas de apoyo formales que plantea el modelo.

La ayuda tangible se utiliza como medio hacia la reconstrucción de las relaciones y transacciones familiares y no como un fin en sí misma. Cuando las familias observan la atención y el compromiso en el/la profesional de ayuda comienzan a evidenciar cambios en las relaciones familiares.

Algunas de las técnicas de intervención utilizadas en la fase intermedia son las siguientes:

\section{Apoyo emocional}

La familia recibe el mensaje: "Estoy para ayudarte, siendo tu compañero/a en el proceso de experimentar otras alternativas a los problemas y necesidades que te plantea tu situación específica de vida". Esta técnica es de fundamental importancia al intervenir en situaciones de crisis en las cuales se afectan los aspectos cognoscitivos, afectivos, somáticos, interpersonales y conductuales de la persona (Slaikeu, 1996).

\section{Mensajes yo}

La intervención terapéutica del/a trabajador/a social puede incluir el fortalecer los patrones de comunicación inter-familiares. Una técnica a utilizarse para dicho propósito puede ser enseñar a los miembros del grupo familiar, especialmente a los/las padres/madres, a utilizar los "mensajes yo". Las metas de dichos mensajes es que la otra persona entienda exactamente la conducta que se desea cambiar sin sentirse intimidada, atacada (Behavioral Science Institute, 1996).

\section{Escuchar activamente}

Esta técnica ayuda al trabajador a escuchar, no sólo el contenido de la comunicación, sino los sentimientos implícitos en la misma (Behavioral Science Institute, 1996).

\section{Refuerzo positivo}

El refuerzo positivo se utiliza como una alternativa al castigo físico. El propósito del refuerzo positivo es aumentar la posibilidad 
de que una conducta cambie. A los padres se les enseña cómo utilizar esta técnica con sus hijos.

\section{Técnicas de negociación y solución de problemas}

Una de las necesidades encontradas en las familias en las cuales ocurre maltrato de menores es poder involucrar a los miembros del grupo familiar en el proceso de negociación y solución de problemas. El/la trabajador/a social enseña esta técnica mediante el modelaje ofrecido en el proceso de ayuda.

\section{Cambiar ideas irracionales}

Otra técnica que el modelo incorpora consiste en ayudar a la persona a combatir ideas irracionales cuya interpretación produce sentimientos de fracaso, desesperanza, frustración y violencia.

\section{Manejo de coraje}

Una de las necesidades encontradas en las familias servidas es el manejar el coraje de forma adecuada. Lo primero que se hace es explicar que el coraje es un sentimiento como otro cualquiera, pero que debemos buscar formas adecuadas para su manejo de manera que no nos hagamos daño ni a nosotros mismos, ni a nuestros familiares $u$ otras personas. Algunos ejemplos que se usan son el termómetro de sentimientos y las tarjetas de crisis (Behavioral Science Institute, 1996).

El modelo incorpora otras técnicas siempre y cuando sean consistentes con sus bases filosóficas y metodológicas. En la etapa intermedia también el/la trabajador/a social puede ofrecer servicios concretos y de intercesoría a los clientes, pero teniendo presente la meta de enseñarles a que puedan resolver los problemas por sí mismos.

Como se indicara anteriormente, la metodología se adapta a las necesidades presentadas por la familia. La intervención puede darse a nivel individual, a nivel de la familia o a nivel grupal. En todos los niveles de intervención el rol de educador del/la trabajador/a social tiene un gran énfasis. Los talleres educativos para madres/padres complementan la intervención a nivel individual y familiar. El grupo pequeño provee una oportunidad excelente para que ocurra el aprendizaje vicario entre los miembros. En este aspecto se incorpora al modelo el concepto de aprendizaje por observación o modelaje según planteado por Bandura (1977). El contenido de los talleres de 
orientación se determina a base de las necesidades comunes de las diferentes familias que participan del proyecto. Pueden contener temas tales como: el cuidado de los infantes, métodos de disciplina y su relación con el desarrollo del auto-concepto y del sentimiento de auto-estima en los/las niños/niñas, comunicación intra-familiar entre otros.

\section{Fase final}

La fase final conlleva el evaluar los logros obtenidos por la familia. El/la trabajador/a social prepara a la familia para el proceso de terminación. Ésta se involucra activamente en el proceso de evaluación. Junto al/la trabajador/a social la familia elabora planes para el futuro. El/la trabajador/a social prepara un informe de progreso y devuelve la situación al Departamento de la Familia con las recomendaciones correspondientes.

\section{Resultados obtenidos}

Los datos expuestos a continuación reflejan los resultados parciales y preliminares del Plan de Servicios Propuestos del Programa AFANA HOMEBUILDERS - Mayagüez.

En el período operacional del año fiscal 1996-97, que comprende del 1 de octubre de 1996 al 30 de septiembre de 1997 se realizaron las siguientes actividades, con estos resultados.

- Se desarrolló con un éxito de 100\% la Primera Fase del Plan que incluía la realización de actividades de divulgación y coordinación con el Departamento de la Familia en todos los niveles administrativos, en adición a la contratación de los trabajadores sociales con maestría y demás oficiales y empleados del Proyecto.

- Se inició la fase de implantación de servicios.

- Se superó el objetivo número uno del Proyecto logrando que el $100 \%$ de las familias participantes identificaran métodos de enfrentamiento positivo a situaciones que conllevan altos niveles de tensión y crisis.

- Se alcanzó el objetivo número dos del Proyecto logrando que el $75 \%$ de las familias participantes establecieran normas y controles parentales definidos y entendibles. 
- Se alcanzó el objetivo número tres del Proyecto logrando que el $75 \%$ de las familias participantes evidenciaran un manejo adecuado de emociones tales como coraje, depresión, ansiedad, frustración y el sentimiento de autoestima.

- Se superó el objetivo número cuatro del Proyecto logrando que el $100 \%$ de las familias participantes pudieran señalar las implicaciones físicas, sociales y emocionales del castigo corporal como método de disciplina.

- Se superó el objetivo número cinco del Proyecto logrando que el $100 \%$ de las familias participantes pudieran identificar métodos de disciplina que no conllevaran el uso de castigo corporal.

- Se superó el objetivo número seis del Proyecto logrando que el $100 \%$ de las familias participantes evidenciaran un aumento en las destrezas de relaciones interpersonales y patrones de comunicación en el seno familiar.

Otros logros

- El Behavioral Science Institute de Seattle, Washington garantizó al Instituto IGODI el permiso para utilizar el nombre "HOMEBUILDERS" para el Proyecto.

- Se estableció la participación de grupos comunitarios representativos de residentes de los residenciales públicos Candelaria, Kennedy, F.D. Roosevelt y Eleanor Roosevelt en actividades educativas y socio-culturales del Proyecto AFANA.

- Se designó al Proyecto AFANA-HOMEBUILDERS Mayagüez como Centro de Práctica Supervisada para estudios Universitarios en Trabajo Social por parte de la Pontificia Universidad Católica de Puerto Rico.

- Se refirieron cuatro estudiantes de Bachillerato en Trabajo Social para realizar práctica supervisada en el Centro de Práctica de AFANA-HOMEBUILDERS.

- Se estableció un comité de voluntarios que ofrecen servicios de apoyo administrativo regularmente.

- Se estableció una red de apoyo empresarial, cívico, religioso 
y cultural al Proyecto AFANA-HOMEBUILDERS.

- Se logró la participación de sesenta y nueve personas representativas de los diversos sectores de la comunidad profesional, industrial, cívica, cultural, religiosa y gubernamental en los Actos de Inauguración del Proyecto y otras actividades socio-educativas con la comunidad.

- Se recibió una Proclama de la Asamblea Municipal de Mayagüez y el Honorable Alcalde reconociendo la apertura del Proyecto AFANA-HOMEBUILDERS en la ciudad de Mayagüez.

- Se diseñó en lenguaje digitalizado una presentación automatizada del Proyecto AFANA-HOMEBUILDERS.

\section{Limitaciones}

La implantación del Proyecto enfrentó algunas limitaciones que se están superando al momento actual. La mayor de éstas fue en el proceso de referidos de las familias. Para lidiar con estas limitaciones se revisó nuevamente dicho procedimiento para garantizar que no se refirieran situaciones de personas que estaban participando de otros proyectos. También se recalcó la importancia de que la participación de las familias fuera voluntaria. Se volvieron a revisar los criterios de selección de las familias y se re-definieron los procedimientos de comunicación entre el Departamento de la Familia y el personal del Proyecto AFANA-HOMEBUILDERS.

\section{Conclusiones}

\section{Impacto en la prestación de servicios y en la política pública}

Definitivamente se vislumbra que como resultado del Proyecto demostrativo surgirá un impacto en la política pública relacionada al manejo del problema de maltrato de menores. Entre los posibles impactos se destacan los siguientes:

- Revisión de la política pública relacionada al reclutamiento de personal para intervenir en situaciones de maltrato de menores. En la actualidad el Departamento de la Familia 
recluta a técnicos cuyo único requisito es que ostenten un bachillerato de una universidad reconocida. Los resultados del proyecto evidencian que para poder implantar el modelo efectivamente es recomendable que se reclute a trabajadores/ as sociales preferiblemente con el grado de maestría. De no tener el grado de maestría podría tener un bachillerato en trabajo social o psicología y recibir un adiestramiento intensivo en el modelo AFANA-HOMEBUILDERS.

- Revisión de la política relacionada al número de situaciones asignadas a cada trabajador/a social. El proyecto ha demostrado que al reducir el número de situaciones aumenta la intensidad y la calidad de la intervención. Esto, a su vez, hace que la duración de la intervención se acorte. Habría que evaluar otros aspectos en la asignación de recursos fiscales. En esta evaluación se recomienda ponderar cuál es el costo social, emocional y económico de la remoción de un niño de su hogar versus el costo de utilizar todos los recursos disponibles para proveer a las familias un servicio de calidad.

- Revisión de la política relacionada al adiestramiento en servicio para el personal. Se recomienda que las personas a cargo de los servicios de protección de menores que no posean preparación en trabajo social reciban adiestramiento especializado en dicho modelo. Existen en Puerto Rico recursos humanos bien preparados académicamente y adiestrados en el modelo, los cuales podrían facilitar esta tarea. Estas personas, al haber participado en los componentes de servicio directo, adiestramiento e investigación social científica representan un caudal de sabiduría unida a una extraordinaria sensibilidad humana.

\section{Implicaciones para la práctica de trabajo social}

Las características y componentes programáticos del proyecto representan unas implicaciones para la práctica de trabajo social. El/ la trabajador/a social debe estar capacitado para transferir un contenido técnico bastante amplio a la prestación de servicios (Tracy, Haapala y Pecora, 1991). 
El conocimiento y las competencias básicas deberán incluir lo siguiente:

- Conocimiento sobre la perspectiva persona en ambiente y reconocimiento de que la familia es la unidad central de la intervención.

- Habilidad para combinar servicios concretos y clínicos y de identificar e intervenir con sistemas de apoyo formales e informales.

- Destrezas para ponderar y utilizar en el proceso de intervención las fortalezas de la familia, de comprometer a las familias en el proceso de establecer las metas del plan de acción.

- Habilidad para comunicarse y cooperar con otras organizaciones de servicio comunitario.

- Conocimiento sobre cómo y cuando enseñar destrezas para ejercer el rol paterno/materno, así como asertividad y otras destrezas de comunicación.

- El reconocimiento de la importancia de la evaluación del trabajo clínico, utilizando diversas metodologías de investigación.

- Conocimiento y destrezas en la intervención en situaciones de crisis.

El Proyecto iniciado representaba un desafío esperanzador en la prestación de servicios para promover el bienestar y la seguridad a las familias servidas. El reto consistía en establecer un modelo innovador, intensivo, asertivo, accesible, flexible y sensitivo culturalmente. El servicio estaba orientado hacia la meta de relevar a las familias de sus presiones y facilitar el desarrollo de un ambiente sano y saludable, dentro del contexto familiar (Fábregas, 1997).

Hemos establecido, junto al Departamento de la Familia, una opción de intervención que provee para fortalecer la unidad familiar y la calidad de vida de la familia. Hemos comenzado este proyecto en la ciudad de Mayagüez, donde, lamentablemente, las estadísticas reflejaban un alto índice de maltrato a menores, pero que, a su vez, 
es una de las menos provista de los servicios de urgencia que necesitaban y merecían estas familias. Sabíamos que los menores en su desarrollo socio-emocional requerían de una relación significativa con sus padres o encargados; también, que la ausencia de destrezas de los padres, en la mayoría de las situaciones era la clave en el deterioro de esas relaciones significativas (Fábregas, 1997).

Sabíamos que, en la mayoría de las veces, donde mejor se desarrollan los menores física y emocionalmente es en su propio hogar, por eso la meta de evitar las remociones innecesarias. El trabajar intensamente con las familias referidas para propiciar la modificación de sus patrones de disciplina y que no recurrieran a estilos maltratantes fue un reto. Estábamos motivados por nuestra misión como trabajadores/as sociales que junto a la visión y objetivos de AFANA-HOMEBUILDERS - Mayagüez, proveyeron la coyuntura del resultado exitoso que ha tenido nuestro Proyecto. El éxito logrado es tanto del equipo de profesionales, como de la Junta de Directores del "Institute for Individual, Group and Organizational Development, Inc." del Departamento de la Familia y, por supuesto, de las familias que acogieron la opción que AFANA-HOMEBUILDERS les presentó y que la incorporaron a su forma de vida (Fábregas, 1997).

\section{Referencias}

Bandura, A. (1977). Social Learning Theory. Englewood Cliffs, New Jersey: Prentice Hall.

Barth, R.P. (1990). Theories guiding home-based intensive family preservation services. En J.K. Whittaker, J. Kinney, E.M. Tracy y C. Booth (Eds.). Reaching high risk families: Intensive family preservation in human services. Hawthorne, NY: Aldine de Gruyter.

Behavioral Science Institute (1997). Fundamentals of Family Preservation Practice. Seattle.

Fábregas, S. (1997). Informe de Progreso del Proyecto AFANA HOMEBUILDERS. Mayagüez: Instituto IGODI.

Fraser, M.W. y Pecora, P.J. (1991). Families in crisis: The impact of intensive family preservation services. Hawthorne, NY: Aldine de Gruyter. 
Jones, M.A. (1991). Conceptualization of outcomes in family preservation services. En D.E. Biegel y K. Wells (eds.). Family preservation services: Research and evaluation. Newbury Park, CA: Sage Press.

Kinney, J.; Haapala, D.; Booth, C. y Leavitt, S. (1990). The HOMEBUILDERS Model. En J. Whittaker, J. Kinney, E. Tracy, y C. Booth (Eds.). Reaching high risk families: Intensive family preservation in human services. Hawthorne, NY: Aldine de Gruyter.

Kinney, J.; Haapala, D. y Booth, C. (1991). Keeping families together: The HOMEBUILDERS model. Hawthorne, NY: Aldine de Gruyter.

Slaikeu, K.A. (1996). Intervención en crisis: Manual para la práctica e investigación. México: El Manual Moderno.

Tracy, E.M. y Whittaker, J.K. (1990). The social network map: Assessing social support in clinical practice. Families in Society, 71(8), 461-470.

Tracy, E.; Haapala, D.; Kinney, J. y Pecora, P. (1991). Intensive family preservation services: An instructional servicebook. Cleveland: Case Western Reserve University.

Whittaker, J.K. (1990). Introduction to the volume. En Whittaker, J.K.; Kinney, J.; Tracy, E.M. y Booth, C. (Eds.). Reaching high risk families: Intensive family preservation in human services. (pp. xiii-xviii). Hawthorne, N. Aldine de Gruyter. 
